\title{
La frontière Lampedusa. Mises en intrigue du sécuritaire et de l'humanitaire
}

The Lampedusa Border. Setting the plot around security and humanitarianism

\section{Paolo Cuttitta}

Traducteur : I'italien par Évelyne Ritaine

\section{Q OpenEdition}

12 Journals

\section{Édition électronique}

URL : http://journals.openedition.org/conflits/19101

DOI : 10.4000/conflits. 19101

ISSN : $1777-5345$

Éditeur :

CECLS - Centre d'études sur les conflits - Liberté et sécurité, L'Harmattan

\section{Édition imprimée}

Date de publication : 31 décembre 2015

Pagination : 99-115

ISBN : 978-2-343-08728-3

ISSN : 1157-996X

Référence électronique

Paolo Cuttitta, "La frontière Lampedusa. Mises en intrigue du sécuritaire et de l'humanitaire », Cultures \& Conflits [En ligne], 99-100 | automne/hiver 2015, mis en ligne le 16 février 2017, consulté le 31 mars 2021. URL : http://journals.openedition.org/conflits/19101; DOI : https://doi.org/10.4000/conflits. 19101 


\section{La frontière Lampedusa. Mises en intrigue du sécuritaire et de I'humanitaire}

\section{Paolo CUTTITTA}

Paolo Cuttitta est chercheur à l'Université libre (VU) d'Amsterdam. Il a publié de nombreux articles et ouvrages sur les politiques migratoires et les contrôles des frontières, parmi lesquels les ouvrages : Lo spettacolo del confine. Lampedusa tra produzione e messa in scena della frontiera, Milan, Mimésis, 2012 ; Segnali di confine. Il controllo dell'immigrazione nel mondo-frontiera, Milan, Mimésis, 2007 ; (dirigé avec Fulvio Vassallo Paleologo) Migrazioni, frontiere, diritti, Naples, Edizioni Scientifici Italiane, 2006. En Français, il a publié "Le monde-frontière. Le contrôle de l'immigration dans l'espace globalisé ", Cultures \& Conflits, 68, 2007 ; "La "frontiérisation" de Lampedusa, comment se construit une frontière ", L'Espace Politique, 25, 2015.

e contrôle de la frontière dans le canal de Sicile peut-il être analysé comme
un «spectacle de la frontière » et l'île de Lampedusa comme la scène privilégiée de la mise en scène de celui-ci ? ${ }^{1}$ L’équation politique-théâtre «fait partie de l'histoire de la politique » 2 , et la «domination spectaculaire » ${ }^{3}$ est capable de manipuler (et donc de construire) l'histoire. L'action politique et son langage peuvent nourrir les processus de construction symbolique de la réalité, et donc du consensus. $\mathrm{Si}$ « la politique est un spectacle ${ }^{4}$ », la frontière se prête particulièrement bien à faire fonction de théâtre. N. De Genova 5 montre comment la frontière entre les États-Unis et le Mexique est le théâtre idéal pour la mise en scène du « spectacle de la frontière » nord-américain. De même, dans les analyses de la frontière méditerranéenne, le recours à la métaphore du spectacle n'est pas rare ${ }^{6}$. Enfin, dans le contexte italien, l'île de

1. Texte traduit de l'Italien par E. Ritaine.

2. Mazzoleni G., La comunicazione politica, Bologna, Il Mulino, 1998, p. 160.

3. Debord G., Commentaires sur la Société du spectacle, Paris, Lebovici, 1988.

4. Edelman M., The Symbolic Uses of Politics, Urbana, University of Illinois Press, 1985, p. 195.

5. De Genova N., "Migrant 'Illegality' and Deportability in Everyday Life", Annual Review of Anthropology, 31, 2002, pp. 419-447.

6. Sossi F., Migrare. Spazi di confinamento e strategie di esistenza, Milano, Il Saggiatore, 2006 ; 
Lampedusa est devenue, durant les vingt dernières années, la frontière par excellence ${ }^{7}$. F. Sossi ${ }^{8}$ a été une des premières à observer comment Lampedusa a été transformée en scène pour le spectacle des politiques de contrôle, mais d'autres aussi ont vu dans l'île sicilienne le théâtre privilégié pour la représentation du spectacle « de la frontière » 9 , « de l'invasion de masse » 10 ou « de l'accueil et de l'urgence $» 11$.

L'urgence est, en effet, un caractère essentiel du régime migratoire actuel, un élément central du «spectacle de la frontière ». En Italie, l'urgence est officielle et permanente depuis 2002, quand l'état d'urgence a été déclaré sur le territoire national, pour toute l'année. Depuis cette date, l'état d'urgence est prorogé tous les ans par de nouveaux décrets. Dans les scénarios de crise, cependant, l'urgence est toujours accompagnée de la dimension humanitaire de l'accueil. Ce n'est pas un hasard si, une fois posée l'idée que l'immigration irrégulière par mer constitue en soi une situation de crise, il a été décidé de donner à la commune de Lampedusa et Linosa la médaille d'or du mérite civil, conférée par le président de la République en 2004. Le motif de cette reconnaissance est que la population lampedusienne « fait preuve envers les immigrés des plus élevés sentiments de solidarité humaine et d'hospitalité » 12.

Le lien entre urgence et accueil correspond aux processus de « sécuritisation » et «d'humanitarisation » bien établis dans la gestion des phénomènes migratoires. Les travaux sur la « sécuritisation» ont montré comment les migrations pouvaient, elles-aussi, être transformées en problème de sécurité ${ }^{13}$. D. Bigo, en particulier, considère la « sécuritisation » comme « une technolo-

Ritaine E., « Dramaturgie de l'intrusion migratoire: Teatro all'italiana », in Martin D.-C. (ed.), L'identité en jeux. Pouvoirs, identifications, mobilisations, Paris, Karthala, 2010, pp. 201-221.

7. Cuttitta P., "La "frontiérisation" de Lampedusa. Comment se construit une frontière ", L'Espace Politique, 25, 2015 [http://espacepolitique.revues.org/3336], consulté le 26 août 2015.

8. Sossi F., "Lampedusa, l'isola che non c'è », in Cuttitta P. et Vassallo Paleologo F. (eds.), Migrazioni, frontiere, diritti, Napoli, Edizioni Scientifiche Italiane, 2006, pp. 251-60.

9. Gatta G., « Le violenze dei salvatori e dei salvati. Scenari lampedusani », Trickster, 10, juin 2011 [http://masterintercultura.dissgea.unipd.it/trickster/doku.php?id=violenza_straniero: gatta_lampedusa], consulté le 26 août 2015.

10. Castronovo A. E., "Dalla "Guerra dei disperati" alle navi galera », in Mannoia M. (ed.), Il silenzio degli altri. Discriminati, esclusi, invisibili, Roma, XL edizioni, 2011, p. 88.

11. Sciurba A., Campi di forza. Percorsi confinati di migranti in Europa, Verona, Ombre Corte, pp. 145-169.

12. Quirinale.it, «Il Presidente Ciampi ha conferito la Medaglia d'Oro al Merito Civile al Comune di Lampedusa e Linosa ", 5 juillet 2004, http://www.quirinale.it/qrnw/statico/expresidenti/ciampi/dinamico/comunicato.asp?id=25038, consulté le 7 juillet 2014.

13. La « sécuritisation » peut être définie comme l'acte de classification qui fait considérer qu'un problème relève de pratiques sécuritaires, cf. Wæver O., Buzan B., Kelstrup M. et Lemaitre P., Identity, Migration and the New Security Agenda in Europe, London, Pinter, 1993 ; Bigo D., "Sécurité et immigration : vers une gouvernementalité par l'inquiétude ? ", Cultures E Conflits, 31-32, 1998 [http://conflits.revues.org/539], consulté le 26 août 2015 ; Huysmans J., 2006, The Politics of Insecurity. Fear, Migration and Asylum in the EU, London-New York, Routledge, 2006. 
gie politique, une modalité de la gouvernementalité contemporaine » basée sur l'inquiétude. La rhétorique humanitaire est aussi un élément essentiel de la « gouvernementalité »14. Selon M. Agier, l'appareil humanitaire peut être aujourd'hui défini, à l'échelle mondiale, comme la main gauche de l'Empire. On peut, en effet, distinguer, « une main qui frappe, l'autre qui soigne » 15. Dans le champ des migrations se vérifie ainsi un cercle vicieux : la " sécuritisation » autorise à considérer le phénomène des migrations comme une urgence ; ce caractère d'urgence légitime l'usage de la main droite pour frapper; en même temps, «l'humanitarisation » de la question impose d'utiliser la main gauche pour soigner ; le fait de devoir soigner, enfin, ne fait que renforcer la notion d'urgence. Et quand il n'y a pas d'urgence, ou qu'elle n'est pas perçue, il est possible de la créer ou de la rendre plus visible. Les États « développent des récits pour expliquer et accomplir leur travail quotidien »; «ils excellent en particulier à jouer (to perform) les crises»16. Les crises, à leur tour, "justifient l'augmentation du pouvoir " ${ }^{17}$. Souvent ce processus « apparaît sur les marges géographiques du territoire national : dans les îles, les aéroports, en mer, et dans les centres de détention offshore »18. L'île de Lampedusa et les mers environnantes le confirment, en effet.

Il faut aussi rappeler que les rhétoriques sécuritaires en matière d'immigration ne se nourrissent pas seulement de la crainte de la criminalité ordinaire et des préoccupations pour l'équilibre du marché du travail : le spectre du terrorisme islamique est lui aussi agité pour justifier des contrôles plus stricts à la frontière méditerranéenne. Selon le ministre de la Défense du second gouvernement Berlusconi, par exemple, l'immigration clandestine « est un phénomène non seulement infiltré par Al Qaeda, mais aussi souvent géré par des terroristes pour faire entrer en Europe des personnes, des armes et de la drogue » ${ }^{19}$. De même le sous-secrétaire d'État à l'Intérieur du second gouvernement Prodi déclare-t-il, en 2006, que les liens « de l'immigration illégale non seulement avec le trafic d'êtres humains, d'armes et de drogue, mais aussi avec le terrorisme international nous obligent à être particulièrement vigilants visà-vis des clandestins venant de la Corne de l'Afrique, comme de l'aire subsaharienne »20. Récemment, le gouverneur de Tobrouk a, lui aussi, tenté d'im-

14. Bigo D., op. cit. ; Fassin D., "Compassion and Repression: The Moral Economy of Immigration Policies in France ", Cultural Anthropology, 20/3, 2005, pp. 362-387 ; Agier M., Gérer les indésirables. Des camps des réfugiés au gouvernement bumanitaire, Paris, Flammarion, 2008.

15. Agier M., « La main gauche de l'Empire ", Multitudes, 11, hiver 2003, [http://www.multitudes.net/La-main-gauche-de-l-Empire/], consulté le 26 août 2015.

16. Mountz A., Seeking Asylum. Human Smuggling and Bureaucracy at the Border, Minneapolis, University of Minnesota Press, 2010, p. xvi.

17. Edelman M., Constructing the Political Spectacle, Chicago/London, University of Chicago Press, 1988, p. 32.

18. Mountz A., op. cit., p. xvii.

19. Martino A., «Discorso del Ministro della Difesa », Roma, Ministero della Difesa, 2004 [http://www.difesa.it/Ministro/Compiti_e_Attivita/Interventi142/2004-11/Pagine/ Discorso_del_Ministro_della_Difesa_250CASD.aspx], consulté le 26 août 2015.

20. Camera dei deputati, "Resoconto stenografico dell’Assemblea. Seduta n. 37 del 19/9/2006 » 
pressioner l'opinion publique européenne en lançant d'improbables avertissements sur la présence de terroristes islamistes dans les barques de migrants, ceci afin d'obtenir un plus grand soutien économique et militaire de l'Italie et de l'Europe ${ }^{21}$.

D’autre part, la rhétorique humanitaire est de plus en plus souvent utilisée pour neutraliser les critiques faites aux politiques de contrôle des frontières, lorsqu'elles sont accusées de violer les droits humains des migrants et de provoquer leur mort : «Les mesures pour contenir la migration par mer sont ainsi présentées comme servant à prévenir à la fois l'immigration illégale et la disparition des migrants durant leurs périlleux voyages » ${ }^{22}$. Cette tendance, nette ces dix dernières années, est explicitée dans divers documents officiels. Le Conseil européen de novembre 2004, non seulement invite « les États membres à améliorer leurs analyses communes des itinéraires de migration, des pratiques en matière de traite des êtres humains et des réseaux criminels actifs dans ce domaine », mais aussi engage " tous les États à intensifier leur coopération en vue de prévenir de nouvelles pertes de vies humaines 23 ». «Contribuer à assurer la protection de la vie des migrants et à leur sauver la vie » est même l'un des objectifs principaux d'Eurosur, le système européen de surveillance des frontières, entré en vigueur en décembre $2013{ }^{24}$. Après le naufrage du 19 avril 2015, au large de la Libye, qui a causé la mort d'environ 700 personnes, une session extraordinaire du Conseil européen a été convoquée, durant laquelle, après avoir établi que « la priorité immédiate est de faire en sorte que plus personne ne meure en mer » ${ }^{25}$, il a été décidé de renforcer la coopération avec les pays d'origine des migrants et les pays de transit. L'objectif principal, toutefois, n'est pas de garantir que les personnes arrivent en toute sécurité, mais bien d'empêcher qu'elles partent, ou de s'assurer qu'elles soient interceptées en mer et reconduites.

Une autre façon de justifier la lutte contre les migrations indésirables est de la présenter comme lutte contre la criminalité organisée des passeurs et des trafiquants. Certes, les crimes contre les migrants commis par ces agents de

[http://www.camera.it/_dati/leg15/lavori/stenografici/sed037/s090.htm], consulté le 26 août 2015.

21. Ansa, "Ministro Tobruk, Isis in arrivo in Italia », 12 mai 2015.

22. Carling J. et Hernández-Carretero M., "Protecting Europe and Protecting Migrants? Strategies for Managing Unauthorised Migration from Africa", The British Journal of Politics and International Relations, 13/1, 2011, p. 45.

23. Conseil de l'Union européenne, Conseil européen de Bruxelles, 4 et 5 novembre 2004. Conclusions de la Présidence, 14292/1/04 REV 1, 2004, pp. 21-25.

24. L'autre objectif est de «détecter, prévenir et combattre l'immigration illégale et la criminalité transfrontalière». Cf. Règlement (UE) n 1052/2013 du Parlement européen et du Conseil du 22 octobre 2013 portant création du système européen de surveillance des frontières (Eurosur), Journal officiel de l'Union européenne, nº2295, 6 novembre 2013, pp. 11-26, Article premier.

25. Conseil de l'Union européenne, Réunion extraordinaire du Conseil européen (23 avril 2015) - déclaration [http://www.consilium.europa.eu/fr/press/press-releases/2015/04/23-specialeuco-statement/], consulté le 26 août 2015. 
voyages irréguliers sont horribles : cela va de la séquestration de personnes au travail forcé, des menaces aux violences physiques (y compris sexuelles), de l'incitation à (et de l'exploitation de) la prostitution aux meurtres. Dans ce cas aussi, la « sécuritisation » du contrôle croise «l'humanitarisation » du contrôle, dans la mesure où les autorités nationales et communautaires présentent leurs politiques et pratiques restrictives comme des mesures humanitaires, afin de les légitimer face à l'opinion publique. En juillet 2003, par exemple, le gouvernement italien de centre-droit présente un nouvel accord avec la Libye comme une manifestation de la volonté des deux parties de mener « une lutte conjointe contre les organisations criminelles (...) qui exploitent impitoyablement les migrants clandestins » 26. Et quand, en décembre 2007, un autre accord avec la Libye est signé par le gouvernement de centre-gauche, le ministre de l'Intérieur explique « qu'il sera maintenant possible (...) de lutter avec une plus grande efficacité contre les trafics, en sauvant de nombreuses vies humaines et en détruisant les bandes criminelles » 27 . Après le naufrage du 3 octobre 2013, près de Lampedusa, qui a causé la mort de 366 personnes ${ }^{28}$, le président de la République italien fait, lui-aussi, de la lutte contre les trafiquants le moyen d'éviter d'autres tragédies ${ }^{29}$. De même, à la suite du naufrage d'avril 2015 près des côtes libyennes, le président du Conseil italien attribue la responsabilité de l'accident aux trafiquants qu'il définit comme « les esclavagistes du XXI ${ }^{\mathrm{e}}$ siècle $» 30$.

De nombreuses analyses sur « l'humanitarisation » des contrôles de l'immigration montrent la complémentarité, plutôt que la contradiction, entre politiques sécuritaires et politiques humanitaires ${ }^{31}$. Ce type d'analyse est ici

26. Ministero dell'Interno, «Comunicato stampa. Firmata dal Ministro dell'Interno Pisanu un'intesa operativa con la Libia sulle modalità pratiche della collaborazione per la lotta all'immigrazione clandestina ", 3 juillet 2003 [http://www1.interno.gov.it/mininterno/site/ $\mathrm{it} / \mathrm{sezioni} / \mathrm{sala} \_s t a m p a / c o m u n i c a t i / c o m u n i c a t o \_353 . h t m l$ ?pageIndex $=11 \&$ year=2003], consulté le 26 août 2015.

27. Ministero dell'Interno, «Immigrazione clandestina: il Ministro dell'Interno Amato firma a Tripoli un accordo per il pattugliamento congiunto della costa libica ", 29 décembre 2007 [http://www1.interno.gov.it/mininterno/export/sites/default/it/sezioni/sala_stampa/ notizie/immigrazione/0871_2007_12_29_ministro_Amato_firma_a_Tripoli_accordo_per_il_ pattugliamento_congiunto.html_1562036151.html], consulté le 26 août 2015.

28. Sur la portée de cet événement et sur les réactions qu'il a suscitées, voir le texte d’Évelyne Ritaine dans ce numéro.

29. Stranieriinitalia.it, « Napolitano: pattugliare le coste da dove partono i migranti », 3 octobre 2013 [http://www.stranieriinitalia.it/attualita/attualita/attualita-sp-754/napolitanoqpattugliare-le-coste-da-cui-partono-i-migrantiq.html], consulté le 26 août 2015.

30. La Repubblica, "Migranti, UE: opzione militare per colpire traffico uomini », 21 avril 2015 [http://www.repubblica.it/esteri/2015/04/21/news/migranti_ue_possibile_operazione_ militare_per_colpire_traffico_essere_umani-112490975/?ref=HREA-1], consulté le 26 août 2015.

31. Walters W., "Foucault and Frontiers. Notes on the Birth of the Humanitarian Border", dans Bröckling U., Krasmann S. et Lemke T. (eds.), Governmentality: Current Issues and Future Challenges, New York, Routledge, 2011, pp. 138-164 ; Williams J. M., "The safety/security nexus and the humanitarianisation of border enforcement", The Geographical Journal, 2014, DOI: 10.1111/geoj.12119 ; Aas K. F. et Gundhus H. O. I., "Policing Humanitarian Borderlands: Frontex, Human Rights and the Precariousness of Life", British Journal of 
repris à l'intérieur d'une vision dramaturgique de la politique ${ }^{32}$. Il s'agit de montrer l'île de Lampedusa comme le théâtre où est donné le «spectacle de la frontière » du contrôle de l'immigration. Les acteurs et les spectateurs sont présentés, selon leurs places et rôles respectifs dans l'architecture de l'espace scénique et dans la dramaturgie de la représentation. On analyse les cinq actes du spectacle, placés dans des conjonctures historiques précises et marqués par des événements spécifiques : les expulsions de 2004-2006, le « modèle Lampedusa » de 2006-2008, la crise des premiers mois de 2009, la parenthèse de «l'immigration zéro » en 2010, la crise de 2011. Ces cinq actes se situent dans la décennie durant laquelle Lampedusa a été transformée, sur le plan opérationnel et sur le plan symbolique, en frontière italienne (et européenne) par excellence ${ }^{33}$.

Chacun de ces actes est analysé selon la réthorique qui le caractérise. Comme cela arrive dans les conflits armés postérieurs à l'époque de la guerre froide, la guerre aux migrations irrégulières est justifiée par le recours à deux registres discursifs différents et apparemment contradictoires : le registre sécuritaire, basé sur la nécessité de garantir l'ordre public et la sécurité face aux menaces qui pourraient venir de l'immigration, et le registre humanitaire, basé sur la nécessité de garantir dignité et droits fondamentaux à toute vie humaine. Sur le théâtre de Lampedusa, les deux registres alternent à l'avant-scène, mais sont en réalité toujours concomittants, étroitement intriqués. Ensemble, ils contribuent à former et à renforcer les politiques et les pratiques de contrôle de l'immigration et des frontières.

\section{Espace scénique, acteurs et spectateurs}

Si le contrôle des frontières maritimes et la gestion des mouvements migratoires peuvent être considérés comme un spectacle politico-médiatique, le principal objectif de cette performance est de présenter, aux yeux des électeurs italiens, ces politiques et ces pratiques comme nécessaires : nécessaires pour protéger non seulement le pays, mais aussi les migrants eux-mêmes.

Les premiers spectateurs du «spectacle de la frontière » sont donc les électeurs italiens. Le message de la pièce est pour eux à la fois anxiogène et tranquillisant, inquiétant et rassurant. Ces citoyens et ces électeurs sont placés à l'orchestre, près de la rampe, comme c'est le cas pour le public populaire dans le théâtre moderne. Parmi eux, cependant, se trouvent aussi de nombreux

Criminology, 2014, DOI: 10.1093/bjc/azu086 ; Pallister-Wilkins P., "The Humanitarian Politics of European Border Policing: Frontex and Border Police in Evros", International Political Sociology, 9/1, 2015, pp. 53-69.

32. Cf. Goffman E., The Presentation of Self in Everyday Life, Garden City, Doubleday Anchor, 1959.

33. Cuttitta P., «La "frontiérisation" de Lampedusa. Comment se construit une frontière », op. cit. 
immigrés : ceux qui sont déjà en Italie, dans des conditions d'irrégularité ou de régularité précaire. À ceux-ci s'adresse surtout le message sécuritaire de certains actes de la pièce, qui doit sonner comme un avertissement de ce qui pourrait leur arriver s'ils ne se comportent pas bien.

Dans les loges, plus éloignées mais en position privilégiée, sont assis deux autres catégories de spectateurs. La première est composée de décideurs européens auxquels l'Italie demande régulièrement un soutien logistique et financier. L'urgence de 2011, comme on le verra, est produite surtout pour tenter d'obtenir l'adoption de mesures communautaires permettant de répartir entre les États membres les personnes qui arrivent sur le territoire italien. La deuxième catégorie est constituée des décideurs politiques des pays d'origine et de transit. L'objectif du spectacle est de les convaincre de participer à la gestion des migrations irrégulières, dans l'intérêt de leur pays et de leurs concitoyens. Ainsi, de spectateurs deviennent-ils co-protagonistes de la pièce, entrant « en scène » bien qu'en demeurant physiquement éloignés. Par exemple, le leader tunisien Z. Ben Ali (qui accepta de collaborer en renforçant les contrôles aux frontières de son pays) ne quittera jamais sa loge en Tunisie. Le dictateur libyen M. Gheddafi, au contraire, empiète sur les places à l'orchestre, durant ses visites en Italie en juin 2009 et août 2010. Le colonel sait qu'il est un partenaire incontournable pour le gouvernement italien, et profite de sa position privilégiée pour transformer ses visites en autant de shows politiques. En 2009, Gheddafi plante ses tentes dans le parc de la Villa Pamphili et se permet de prononcer des discours aussi théâtraux que provocateurs ${ }^{34}$. En 2010, il donne un spectacle équestre composé de trente cavaliers berbères venus de Libye et organise une rencontre avec 500 jeunes femmes, recrutées par une agence d'hôtesses, lors de laquelle il formule l'espoir que « l'Islam devienne la religion de l'Europe » 35 . Puis, il passe à la menace de faire virer au «noir » le vieux continent, en l'inondant de migrants africains ${ }^{36}$.

Ces intrusions, conformes aux expérimentations du théâtre d'avant-garde $\mathrm{du} \mathrm{XX}^{\mathrm{e}}$ siècle, contestent non seulement la distribution rigide des rôles, en impliquant les spectateurs dans le spectacle et en les transformant en protagonistes, mais aussi les divisions très nettes de l'espace scénique (notamment la

34. Par exemple, la comparaison entre les États-Unis de Reagan et l'Al-Qaeda de Bin Laden, les critiques contre la guerre en Irak, mais aussi contre le "partitisme » des pays occidentaux, considéré comme « un avortement de la démocratie » : "Gheddafi: "Usa come Bin Laden. Partitismo aborto della democrazia” ", La Repubblica, 11 juin 2009 [http://www.repubblica.it/2009/06/sezioni/esteri/gheddafi-italia/gheddafi-senato/gheddafisenato.html], consulté le 26 août 2015.

35. La Repubblica, "Gheddafi show nella capitale. "Islam diventi religione Europa” ", 29 août 2010 [http://www.repubblica.it/politica/2010/08/29/news/la_visita_di_gheddafi-6588415/ index.html?ref=search], consulté le 26 août 2015.

36. Corriere della Sera, "I limiti invalicabili per il buon nome del paese », 31 août 2010 [http://www.corriere.it/editoriali/10_agosto_31/i-limiti-invalicabili-per-il-buon-nome-delpaese-franco-venturini_1f506028-b4bd-11df-8e04-00144f02aabe.shtml], consulté le 26 août 2015. 
séparation entre espace de représentation et espace de jouissance du spectacle). Malgré tout, la scène principale de la pièce demeure Lampedusa. Sur cette scène, les acteurs principaux rencontrent une autre partie du public : les migrants. Au moins dans un premier temps, quand ils se trouvent dans leur pays d'origine ou dans un pays de transit, les migrants potentiels sont-ils, en fait, des spectateurs : le sont aussi bien ceux qui sont déjà en train de penser à la traversée du canal de Sicile, que ceux qui pourraient y songer dans le futur. Ceux-ci n'occupent pas les fauteuils des loges, réservés aux leaders politiques de leur pays, mais les sièges inconfortables des galeries, dans les recoins éloignés et sombres du théâtre. Même de là, cependant, ils parviennent à voir ce qui arrive, de bon ou de mauvais, à qui tente de traverser. Et malgré cela (ou à cause de cela), souvent ils décident de partir. Une fois arrivés à Lampedusa, sur la scène principale, leur rôle change et ils deviennent la matière première du spectacle. Leur position et leur fonction sur la scène peut changer selon les actes de la représentation. Dans le premier acte, ils sont si dispersés qu'ils en deviennent invisibles : ils rappellent ces éléments scéniques, que, bien qu'essentiels à la compréhension du spectacle, les scénographies du théâtre expressioniste préférent omettre, en se limitant à les suggérer symboliquement. À l'inverse, dans les troisième et cinquième actes, les immigrés font irruption sur la scène, jusqu'à la remplir littéralement de leurs corps. Le quatrième acte montre comment ils peuvent être les maîtres de la scène, même quand ils sont non seulement invisibles mais aussi physiquement absents. Dans le second acte enfin, leur présence n'est que discrétement perçue car elle se mêle à celle de tant d'autres acteurs, institutionnels ou non.

Le « spectacle de la frontière » mis en scène à Lampedusa est donc caractérisé par un double registre narratif, qui oscille entre urgence sécuritaire et scrupule humanitaire, entre rhétorique de la frontière «dure » et rhétorique de la frontière « humaine 37 .

\section{Premier acte (Fermeté)}

Le premier acte est placé sous le signe d'une dureté abrupte. Il s'agit de la série de déportations exécutées entre octobre 2004 et mars 2006. Plus de 2000 migrants ont été expulsés directement de Lampedusa vers la Libye, pays d'où ils s'étaient embarqués. Le spectacle est sobre et essentiel. Le plus important est le message envoyé au public, qui peut être résumé ainsi : «Nous défendons nos frontières, nous renvoyons les clandestins là d'où ils sont venus ». Aucune scénographie particulière n’est nécessaire : la simple actualisation quotidienne

37. Il arrive la même chose dans d'autres lieux frontières comme la Méditerranée du sud-est (Bojadžijev M. et Karakayalı S., " Autonomie der Migration. 10 Thesen zu einer Methode », in Transit Migration Forschungsgruppe (Hrsg.), Turbulente Ränder. Neue Perspektiven auf Migration an den Rändern Europas, Bielefeld, Transcript, 2007, pp. 203-209) et l'Australie (Neilson B., "Between governance and sovereignty: remaking the borderscape to Australia's north”, Local-Global, 8, 2010, pp. 124-140). 
des nombres de personnes expulsées vers la Libye suffit. En revanche, dans la mesure où le gouvernement est conscient d'agir en violation (ou au moins aux limites) de la loi, il cherche à éviter que trop de détails soient rendus publics.

F. Sossi remarque que, dans cette période, les médias montrent seulement des images neutres du port, de la côte, et quelquefois les visages des migrants à leur arrivée, mais aucune image des opérations de reconduite ${ }^{38}$. Les premiers vols d'expulsion ont lieu de façon inattendue, sans préavis. La population de Lampedusa, elle-même, ne voit pas ce qui se passe, et l'attention extérieure à l'égard de l'île n'est pas comparable à ce qu'elle sera en 2009 et 2011, aux troisième et cinquième actes. Les expulsions se poursuivent dans l'ombre, et souvent sans qu'il en soit question. Disparaissent donc, aussi, les données statistiques à ce sujet.

Durant ces années, la gestion des arrivées est encore aux mains des seules autorités d'État et de l'entreprise gestionnaire du centre de détention. D'autres acteurs - à commencer par les organisations humanitaires - en sont exclus, au point que l'accès au centre est même interdit au Haut commissariat des Nations-Unies pour les réfugiés (HCR). Durant ce premier acte, donc, la rhétorique sécuritaire est largement dominante.

\section{Deuxième acte (Humanité)}

La rhétorique de la frontière "humaine " ne réussit à s'affirmer qu'après la victoire de la coalition de centre-gauche (R. Prodi), en avril 2006. Cependant, avant les élections il y avait déjà eu une période de transition durant laquelle les deux rhétoriques commencèrent à se mélanger. Après les lourdes critiques que le gouvernement a subi pour les expulsions vers la Libye, celui-ci décide de réduire nettement ces pratiques (en 2006, il n'y a qu'un vol de Lampedusa vers Tripoli). De plus en février 2006, il modifie le statut du centre de détention de Lampedusa: de centre de détention temporaire (centro dipermanenza temporanea: $\mathrm{Cpt}$ ), il devient centre de premier secours et d'accueil (centro di primo soccorso e accoglienza: Cpsa). À partir de cette date, les migrants ne devront rester à Lampedusa que pour les premiers soins médicaux et pour les procédures d'identification, avant d'être transférés sur le continent. Pour ce faire, le gouvernement lance le projet Praesidium, co-financé par l'Union européenne (UE), auquel participent le Haut Commissariat des Nations unies pour les Réfugiés (HCR), l'Organisation internationale pour les migrations (OIM), et la Croix rouge italienne ${ }^{39}$ : durant leur bref séjour sur l'île, les migrants doivent être soumis à un «screening ", effectué par le gouvernement italien avec ces organisations.

38. Sossi F., "Lampedusa, l'isola che non c'è », op. cit.

39. Projet auquel participe aussi l'ONG Save the children à partir de 2008. 
Entre 2006 et 2008, cette coopération entre les autorités de l'État et les organisations internationales et non gouvernementales s'amplifie, tant dans le cadre du projet Praesidium qu'à l'extérieur de celui-ci, avec l'implication de Médecins sans frontières et de l'Ordre de Malte. En fait, l'implication des ONG garantit que le système soit à la fois efficient et respectueux des droits, non seulement dur et inflexible, mais aussi humain et charitable.

En novembre 2006, A. Guterres, Haut commissaire des Nations unies pour les Réfugiés, déclare, après une visite du centre, "qu'il y a beaucoup de progrès, que la qualité de la vie y a vraiment changé, que la situation est en train d'évoluer 40 ». En janvier 2008, c'est au tour de l'OIM de déclarer que beaucoup de problèmes du centre de Lampedusa sont désormais résolus, au point que « le centre peut être un modèle pour d'autres pays » ${ }^{41}$. Certes, le fait que l'OIM et le HCR louent leur propre travail pourrait être attribué à une capacité d'autocritique insuffisante de la part des deux organisations, toutes deux présentes à l'intérieur du centre. Cependant, le Conseil européen enregistre lui-aussi des progrès significatifs. Sans cacher «sa préoccupation quant au statut légal du centre d'accueil », dans la mesure où « manque la base légale pour la détention de personnes », le rédacteur du rapport sur les flux migratoires arrivant par mer en Europe méridionale « félicite les autorités italiennes pour les progrés accomplis et pour l'approche intégrée adoptée dans la gestion du centre $\gg 42$.

C'est pourtant dans ce cadre humanitaire que se réaffirme la dureté des politiques italiennes. Alors que le gouvernement Prodi renforce la coopération avec le régime de Gheddafi, de façon à empêcher les migrants d'embarquer à partir des côtes libyennes (les faisant de fait finir dans les centres de détention libyens, dans des conditions de vie atroces, à la merci des abus et de la violence de la police locale ${ }^{43}$ ); alors que ce même gouvernement signe avec la Libye, en décembre 2007, un accord de coopération de police (qui permettra au gouvernement suivant, celui de S. Berlusconi, de mettre en place en 2009 des expulsions illégitimes dans les eaux internationales) : dans ce même temps, la face interne du régime frontalier italien - la scène de Lampedusa - demeure immaculée, grâce à la fin des expulsions et au respect des droits humains sur l'île.

40. Ansa, «Immigrazione: Lampedusa; Unhcr, vita in Cpt è migliorata. Alto Commissario Guterres valuta condizioni », 16 novembre 2006.

41. Ansa, "Immigrazione: Esperto OIM, centro Lampedusa modello da seguire », 18 janvier 2008.

42. Council of Europe, «Europe's 'boat people': mixed migration flows by sea into southern Europe ", Parliamentary Assembly Doc. 11688, 11 juillet 2008, p. 17 [ht t p://assembly.coe.int/nw/xml/XRef/X2H-Xref-View HTML.asp? FileID=11980\&lang=en], consulté le 26 août 2015.

43. Human Rights Watch, "Pushed Back, Pushed Around. Italy's Forced Return of Boat Migrants and Asylum Seekers, Libya's Mistreatment of Migrants and Asylum Seekers ", septembre 2009 [http://www.hrw.org/sites/default/files/reports/italy0909web_0.pdf], consulté le 26 août 2015. 
Un nouvel acte commence à la fin 2008 pour se terminer en mars 2009. Le récit de la frontière «dure » reconquiert la scène de Lampedusa qaund le nouveau gouvernement (S. Berlusconi) décide de ralentir les transferts depuis l'île, précisément au moment où les arrivées s'y accroissent, avec l'intention évidente d'y créer, artificiellement, une situation d'urgence. Aussi le centre de Lampedusa se remplit-il toujours plus. Il a une capacité de 381 places en temps normal, de 804 en situation d'urgence. Or, dans le centre, se trouvent 1560 résidents le 11 novembre 2008, et 1572 résidents le 28 décembre. Les migrants sont pour la plupart tunisiens, mais les Égyptiens et les sub-sahariens sont aussi nombreux. Le 30 décembre, le gouvernement annonce que désormais quiconque arrivera à Lampedusa sera détenu sur l'île pour un temps indéterminé, jusqu’à son rapatriement ; les demandes d'asile devront aussi être examinées sur l'île.

Tout ceci survient alors que le Sénat est en train de discuter un projet de loi qui prévoit de prolonger les temps maximums de maintien dans les centres de détention de 60 jours à 18 mois. Il semble que le gouvernement ait choisi Lampedusa pour les répétitions de ce nouvel acte, bien que le centre de l'île ne soit plus un centre de détention, mais, depuis février 2006, un centre de premier secours et d'accueil. De novembre 2008 à mars 2009, des centaines de migrants y sont maintenus en détention bien au-delà des 60 jours légaux. Le mécontentement monte, non seulement parmi les migrants, mais aussi parmi les habitants de l'île. En janvier 2009, le gouvernement annonce la construction d'une seconde structure, déchaînant les protestations des habitants qui ne veulent pas voir leur île transformée en prison. Le 23 janvier, ils organisent une manifestation de protestation. Le même jour, les surveillants laissent «s'échapper » une grande partie des 1840 migrants détenus dans le centre. Beaucoup rejoignent la manifestation des habitants. Les images de centaines de migrants dans les rues de Lampedusa sont reprises par tous les journaux télévisés. En réponse, les autorités envoient des centaines de policiers, de carabiniers et de membres de la police financière (Guardia di Finanza).

Le 5 février, de façon surprenante, le Sénat rejette la prolongation des temps de détention des migrants irréguliers. Entre-temps, cependant, l’Italie et la Tunisie ont signé un accord pour faciliter l'identification et le rapatriement des Tunisiens. Le 3 février commencent les premières reconduites vers la Tunisie et la situation devient explosive. Le 17 février, les migrants organisent une grève de la faim pour protester contre la menace de rapatriement, et le jour suivant ils déclenchent un incendie. Le centre est en partie détruit, ce qui entraîne le transfert de centaines de migrants sur le continent, en contradiction avec les déclarations intransigeantes du gouvernement. L'importance des dégâts et l'aspect spectaculaire de l'incendie contribuent non seulement à ren- 
forcer l'idée d'état d'urgence, mais aussi préparent à la criminalisation ultérieure des migrants. Dans ce climat, le gouvernement, seulement deux jours après l'incendie, étend par décret la durée maximale de détention à 6 mois.

En fait, certaines des déclarations du gouvernement dans cet acte du spectacle restent lettre morte. À l'exception d'un nombre ridicule de demandes d'asile examinées sur l'île immédiatement après l'annonce gouvernementale, toutes les autres sont présentées et examinées plus tard, sur le continent. De plus, des centaines de soi-disant « migrants économiques » sont transférés en Sicile ou dans d'autres régions, soit pour être rapatriés à partir de là, soit pour être laissés en liberté (parce que les centres de détention italiens sont pleins, ou parce que le temps légal de détention est atteint). Mais le plus important est que le ton de la «dureté » l'emporte : dureté de l'attitude intransigeante du gouvernement, dureté de la situation engendrée à Lampedusa. En effet, les conditions exceptionnelles et dramatiques créées sur l'île servent de moyen de pression sur les autorités tunisiennes, jusque-là peu enclines à accepter les rapatriements ; elles servent aussi à écrire le scénario de l'invasion et de l'urgence nécessaire pour justifier l'adoption de mesures restrictives et répressives. La première de ces mesures est l'allongement du temps maximal de rétention. La dernière sera la reprise des expulsions collectives vers la Libye, quelques mois plus tard. Le troisième acte se conclut le 18 mars 2009 quand le ministre de l'Intérieur R. Maroni annonce qu'à partir du mois de mai commenceront « les patrouilles conjointes (italo-libyennes) devant les côtes libyennes ", et qu'ainsi "l'affluence des clandestins sera drastiquement réduite, voire supprimée $» 44$.

\section{Quatrième acte (Immigration zéro)}

Le quatrième acte commence plus d'une année après le début de la nouvelle vague de reconduites. À la différence de celles des années 2004-2006 (qui ne concernaient que des personnes déjà présentes sur le territoire italien), les reconduites effectuées par le gouvernement Berlusconi vers la Libye à partir de 2009 ont lieu directement depuis les eaux internationales, dans le cadre des patrouilles conjointes italo-libyennes. Les migrants ne sont conduits à Lampedusa que si l'un d'eux a besoin de soins immédiats. Dans ce cas, après avoir débarqué les malades, les vedettes italiennes font immédiatement route vers la Libye. Lampedusa voit ainsi confirmé son rôle de scène théatrâle : non pas tant parce que les vedettes de reconduite y font des passages rapides, ni parce que l'île est la base opérationnelle des moyens d'expulsion, mais parce que l'île est peu à peu vidée de migrants. En effet, les patrouilles dans les eaux territoriales libyennes (qui limitent les départs) et les expulsions depuis les eaux internationales (qui colmatent les brèches qui peuvent appparaitre ça et

44. Camera dei deputati, « Resoconto stenografico dell’Assemblea. Seduta n. 148 di mercoledì 18 marzo 2009 " [http://leg16.camera.it/410?idSeduta=0148\&tipo=stenografico\#sed0148. stenografico.tit00050.sub00050.int00080], consulté le 26 août 2015. 
là) réduisent presque totalement le nombre d'embarcations qui parviennent dans les eaux territoriales italiennes.

En août 2010, le ministre de l'Intérieur annonce que le nombre des migrants arrivés à Lampedusa s'est réduit de $98 \%$ par rapport à l'année précédente. Lampedusa devient ainsi la scène où se déroule le show de "l'immigration zéro » : une nouvelle variation de la rhétorique de la frontière «dure ». Le centre de détention est alors fermé, cependant que les rares migrants qui réussissent à rejoindre les eaux territoriales italiennes ne sont plus conduits à Lampedusa, mais dans d'autres ports siciliens, pour ne pas troubler le déroulement de la pièce. Les rares migrants qui arrivent sur l'île sont, quant à eux, rapidement transférés en Sicile, pour que le centre de Lampedusa puisse demeurer fermé, sous le signe de «l'immigration zéro ».

\section{Cinquième acte (Urgence bis)}

Le cinquième acte aussi participe de la rhétorique de la frontière "dure». Dans ce cas, cependant, ce récit se mêle à celui de la «crise humanitaire ». Les arrivées en provenance de Tunisie augmentent pour la première fois depuis des années, à la fin de 2010. Le centre de Lampedusa reste fermé pourtant : plutôt que de ruiner la scène où se déroule le show de « l'immigration zéro ", les autorités choisissent de loger les migrants dans des hôtels. À partir de janvier 2011 et de la chute du régime de Ben Ali, les arrivées se multiplient. Les migrants tunisiens arrivent par leurs propres moyens ou bien sont débarqués par les vedettes italiennes : les autorités comprennent alors que le scénario «immigration zéro » n'est plus tenable et qu'il faut changer de registre narratif. Le gouvernement, cependant, ne décide ni de rouvrir le centre, ni de transférer les migrants ailleurs en Italie. Le ministre de l'Intérieur R. Maroni se limite à insister sur l'aspect sécuritaire de l'urgence. Il déclare d'abord: "Quand je lis qu'une prison de haute sécurité égyptienne a été incendiée et que les prisonniers se sont échappés, je prévois quels effets pourrait avoir cette situation " 45 , allusion au risque que ces criminels évadés puissent débarquer en Italie. Dix jours plus tard, au sujet de la Tunisie, il fait l'hypothèse que, parmi les personnes fuyant le pays, il puisse y avoir « aussi des personnes infiltrées par les organisations terroristes $" 46$.

Le 12 février, environ 4000 migrants sont contraints à dormir dans les rues de Lampedusa. Le jour suivant, le gouvernement déclare l'état d'urgence humanitaire sur tout le territoire ${ }^{47}$. Alors seulement commencent, lentement,

45. Corriere della Sera, «Maroni: condivido i timori di Napolitano. Giovedì decisivo, federalismo o elezioni », 31 janvier 2011 [http://rassegna.governo.it/testo.asp?d=55614519], consulté le 26 août 2015.

46. Corriere della Sera, Lampedusa, nuova ondata di immigrati. Via i trasferimenti, ma c'è rischio collasso, 11 février 2011 [http://www.corriere.it/cronache/11_febbraio_11/lampedusaimmigrati-sbarco_75f8c7fo-35c1-11e0-9a90-00144f486ba6_print.html], consulté le 26 août 2015. 
les transferts vers la Sicile, et alors seulement le centre de Lampedusa est rouvert. Il y a encore des milliers de personnes sans abri. Ainsi, avant même d'être déclaré, l'état d'urgence humanitaire a été créé par le gouvernement, comme avait été créée la crise de 2009 : en évitant de transférer les migrants. Cette fois, cependant, l'île tout entière a été transformée en camp à ciel ouvert, et ce pour une longue période : fin mars 2011, il y a encore 6200 migrants sur l'île, soit plus que d'habitants à Lampedusa (5 800 habitants). Les habitants s'efforcent de porter assistance aux migrants sans abri. L'approvisionnement en nourriture devient problématique. Les transferts continuent au ralenti, pourtant.

L'objectif de l'urgence humanitaire (provoquée puis déclarée par le gouvernement) est d'obtenir de l'UE l'adoption des mesures de protection temporaire prévues, en cas d'afflux massif de personnes déplacées, par une directive communautaire 48 : de cette façon, les migrants seraient dispersés entre les différents États membres. Il apparaît très vite que l'UE ne prendra pas une telle décision : le gouvernement décide alors d'agir unilatéralement et adopte une mesure nationale de protection transitoire. Le 5 avril, après la signature d'un nouvel accord avec le gouvernement de transition tunisien, le président $\mathrm{du}$ Conseil S. Berlusconi promulgue un décret qui prévoit que toute personne arrivée sur le territoire avant cette date va recevoir un permis de séjour pour motif humanitaire, valable six mois. Les migrants qui arriveront après cette date seront au contraire immédiatement expulsés, quel que soit leur pays d'origine. Les premiers rapatriements ont lieu immédiatement et les arrivées de Tunisiens se réduisent notoirement dans les mois suivants. Les bénéficiaires de la protection temporaire, eux, sont finalement libres : libres de se déplacer en Italie comme de franchir les frontières terrestres avec les autres pays de l'espace Schengen. Malgré les tensions avec la France, qui rétablit en partie les contrôles à la frontière, de nombreux Tunisiens atteignent leur objectif : utiliser l'Italie comme pays de transit. De cette façon, l'objectif du gouvernement, réduire le nombre de migrants présents sur le territoire, est lui aussi en partie atteint. Ainsi cet acte est-il encore dominé par le récit sécuritaire de la frontière «dure ». Cependant, ce n'est qu'en introduisant l'élément humanitaire dans le récit sécuritaire de la frontière, ce n'est qu'en navigant « entre un cadre humanitaire et un cadre sécuritaire ${ }^{49}$ que le gouvernement parvient à renforcer la rhétorique de la frontière «dure».

47. Il ne s'agit pas du même type d'état d'urgence que celui décrété face aux débarquements en 2002 puis continuellement prorogé : celui-ci a un caractère générique. L’urgence décrétée en 2011 a, elle, un caractère spécifiquement humanitaire.

48. Directive 2001/55/CE du Conseil du 20 juillet 2001 relative à des normes minimales pour l'octroi d'une protection temporaire en cas d'afflux massif de personnes déplacées et à des mesures tendant à assurer un équilibre entre les efforts consentis par les États membres pour accueillir ces personnes et supporter les conséquences de cet accueil, Journal officiel de l'Union européenne, $\mathrm{n}^{\circ} \mathrm{L} 212,7$ août 2001, pp. 12-23.

49. Campesi G., The Arab Spring and the Crisis of the European Border Regime. Manufacturing Emergency in the Lampedusa crisis, EUI Working Paper RSCAS, 59, 2011, p. 9. 
L'urgence du cinquième acte ne se termine pas en avril 2011. Après les arrivées des Tunisiens viennent celles, aussi nombreuses, provoquées par la guerre en Libye. En été, les arrivées de Tunisiens reprennent aussi. Le 18 septembre, 1500 personnes sont enfermées dans le centre de Lampedusa. Le 20, un incendie détruit une grande partie du bâtiment et le lendemain un groupe de migrants, exaspérés par la détention prolongée et par la perspective du rapatriement, menace de faire exploser des bonbonnes de gaz près du port. Ils provoquent la réaction violente de lampedusiens, eux aussi exaspérés par le trop long état d'urgence, qui agressent les migrants. Les jours suivants, tous les migrants sont éloignés de l'île, placés à bord de navires transformés en centres de détention itinérants, puis transférés dans d'autres structures italiennes ${ }^{50}$, tandis que le centre est fermé et l'île déclarée " port non sûr » : personne ne devra plus y être conduit ${ }^{51}$.

\section{Conclusion}

L'analyse des politiques de contrôle de l'immigration dans le canal de Sicile montre que celles-ci sont marquées par la combinaison de deux logiques d'intervention et de deux registres narratifs, apparemment opposés, mais en fait complémentaires : la logique et le registre de la sécurité qui se réfèrent à la nécessité de protéger les frontières de la menace présumée de l'immigration irrégulière ; la logique et le registre de l'humanité, qui se réfèrent à la nécessité de sauver des vies et de respecter les droits humains. La réflexion s'est concentrée sur la période historique (la décennie qui s'est achevée avec les " printemps arabes » de 2011) qui a vu Lampedusa devenir la frontière italienne et européenne par excellence.

L'analyse des registres narratifs du «spectacle de la frontière » montre que la rhétorique humanitaire trouve son expression maximale entre 2006 et 2008, durant le second gouvernement Prodi. Les scènes qui expriment le mieux la rhétorique de la frontière « dure » sont, elles, distribuées entre plusieurs actes, montés durant les différents gouvernements Berlusconi. On voit ainsi qu'« humanitaire » et « sécuritaire » sont les deux faces d'une même médaille : les deux registres se soutiennent et concourent à l'écriture de la pièce; ils contribuent à construire le régime de contrôle des frontières maritimes.

Les événements récents le confirment, de même qu'ils confirment le rôle de Lampedusa comme scène privilégiée, du moins jusqu'à un certain moment. C'est à Lampedusa que le pape François a souhaité se rendre, en juillet 2013, pour dénoncer l'indifférence croissante envers les droits et la vie même des migrants. Un peu plus tard, le 3 octobre, la mort de 366 personnes à quelques

50. Castronovo A. E., op. cit.; Vassallo Paleologo F., Diritti sotto sequestro, Roma, Aracne, 2012.

51. Le centre sera en partie rouvert durant l'été 2012, puis de nouveau fermé en décembre 2013 pour des travaux, qui sont terminés en octobre 2014, date de la réouverture du centre. 
centaines de mètres de l'île a réveillé la compassion et le sens de l'humanité dans toute l'Europe ${ }^{52}$. La réponse des institutions italiennes et européennes a été, encore une fois, le renforcement des mesures de lutte contre les migrations, à commencer par la collaboration avec les pays nord-africains, pour empêcher les migrants d'embarquer. À la lumière de la réflexion conduite ici, on ne peut s'étonner qu'un tel objectif soit poursuivi au travers d'une plus grande légitimation humanitaire de Frontex ${ }^{53}$, du lancement du système de surveillance Eurosur (dont un des objectifs affichés est de sauver des vies humaines), et du renforcement par les autorités italiennes des habituelles activités de patrouille en Méditerranée, avec une nouvelle opération militaire : Mare Nostrum, présentée comme une mission humanitaire destinée à sauver des vies, mais en même temps partie intégrante de l'appareil de contrôle des migrations irrégulières ${ }^{54}$.

En 2013, cependant, la centralité de Lampedusa comme scène du « spectacle de la frontière » commence à diminuer. L'urgence provoquée sur l'île en 2011 a été si insupportable qu'il n'est pas envisageable qu'elle puisse se répéter. Les autorités italiennes ont, en conséquence, décidé, face à l'intensification des arrivées par mer (lors de l'aggravation de la crise en Syrie), de disperser les personnes interceptées entre différents ports, évitant ainsi que Lampedusa soit à nouveau étouffée. Le drame du 3 octobre, qui ramène violemment l'île sous les projecteurs, en la remplissant de cadavres et de survivants, intervient comme un élément perturbateur du spectacle, comme un « accident » 55, comme l'obscène ${ }^{56}$ qui s’impose sur scène. Cet événement imprévu contribue d'autant plus à confirmer le choix de disperser les personnes dans différents ports que le centre d'accueil de l'île est fermé pour une année. Lampedusa ne redevient ainsi un lieu de débarquement fonctionnant à plein régime qu'en 2015.

L'élargissement de la scène au niveau national s'accompagne, plus récemment, de la forte augmentation du nombre de personnes qui se mettent en route vers l'Europe, ainsi que de l'évolution des routes empruntées, qui passent de plus en plus par la mer Égée et la péninsule des Balkans. Pendant que Lampedusa perd une partie de sa centralité opérationnelle (mais pas sa centra-

52. Pour cette période, voir le texte d’Évelyne Ritaine dans ce numéro.

53. Agence européenne pour la gestion de la coopération opérationnelle aux frontières extérieures. Cf. Aas K. F. et Gundhus H. O. I., op. cit. ; Pallister-Wilkins P., op. cit.

54. Cuttitta P., "From the Cap Anamur to Mare Nostrum. Humanitarianism and Migration Controls at the EU's Maritime Borders", in Matera C. et Taylor A. (eds.), The Common European Asylum System and Human Rights: Enhancing Protection in Times of Emergencies, CLEER Working Papers, 7, 2014, pp. 21-37 ; Tazzioli M., "The Desultory Politics of Mobility and the Humanitarian-Military Border in the Mediterranean. Mare Nostrum Beyond the Sea", REMHU: Revista Interdisciplinar da Mobilidade Humana, 23/44, 2015, pp. 61-82.

55. Cf. Goffman E., op. cit.

56. Littérallement : qui doit rester hors de la scène, cf. De Genova N., "Spectacles of migrant 'illegality': the scene of exclusion, the obscene of inclusion", Ethnic and Racial Studies, 36/7, pp. 1180-1198. 
lité symbolique), le « spectacle de la frontière » européen se propage jusqu’à occuper non pas une seule scène locale, non pas une seule scène nationale, mais tout l'édifice théâtral qu'est le continent européen. Dans ce cadre, les logiques et les réthoriques décrites pour l'île de Lampedusa s'affirment de la même façon. La cohérence et la tenue du spectacle semblent, cependant, aujourd'hui mises en question par l'incapacité à en contrôler le scénario. L'augmentation permanente des arrivées et surtout l'irruption de la mort de masse, sa répétition fréquente depuis le drame du 3 octobre 2013, sa propagation sur plusieurs lieux géographiques (sur plusieurs «scènes ») rendent toujours plus instable l'équilibre entre les diverses logiques et réthoriques en jeu.

\section{Boîte noire méthodologique}

Ce texte reprend, en l'actualisant et en le développant, le chapitre conclusif de mon livre : Lo spettacolo del confine. Lampedusa tra produzione e messa in scena della frontiera, Milan, Mimesis, 2012.

La recherche, menée entre 2005 et 2015, a été développée au travers de l'analyse de documents (articles de presse, communiqués de presse, rapports d'ONG, documents institutionnels, etc.). De plus, dans cette période, plusieurs séquences d'enquête de terrain se sont déroulées à Lampedusa. Elles ont permis, notamment, de réaliser des entretiens semidirectifs et non directifs avec des agents de la frontière : membres d'institutions d'État comme les gardes-côtes, les forces armées, les pompiers, administrateurs locaux, travailleurs intervenant dans les institutions chargées de l'accueil, membres d'associations de volontaires ou d'associations d'activistes; avec des migrants ; avec des habitants de l'île.

Avec le temps, les conditions de la recherche sur le terrain à Lampedusa ont changé. Le processus de «frontiérisation » de l'île, décrit dans l'article, a eu pour conséquence qu'une partie de la population et des opérateurs de la frontière ont développé un sentiment de saturation face à ceux qui viennent de l'extérieur pour recueillir des informations et mener des entretiens.

Ceci a pu se traduire, parfois, par un manque de disponibilité ou une certaine intolérance à l'égard de l'interlocuteur. D'autres fois, cela a entraîné des réponses standardisées et mécaniques, qui visent à fournir les réponses que l'interlocuteur est supposé attendre, ou à éluder le sens de la question pour s'épargner l'effort d'élaborer une réponse. De plus, dans le cas des représentants de l'autorité et des opérateurs de l'accueil, la standardisation des réponses correspond souvent à la volonté d'éviter des questions politiquement dérangeantes.

Pour remédier, au moins en partie, à ces inconvénients, il a été nécessaire de développer, avec le temps, des rapports privilégiés avec certains enquêtés et de se distinguer comme étant un interlocuteur différent et plus qualifié que les autres. 